\title{
Effect of triplet attractions on the phase diagram of suspensions of charged colloids
}

\author{
Antti-Pekka Hynninen ${ }^{1}$, Marjolein Dijkstra ${ }^{1}$ and René van Roij ${ }^{2}$ \\ ${ }^{1}$ Debye Institute, Soft Condensed Matter Physics, Utrecht University, Princetonplein 5, \\ 3584 CC Utrecht, The Netherlands \\ ${ }^{2}$ Institute for Theoretical Physics, Utrecht University, Leuvenlaan 4, 3584 CE Utrecht, \\ The Netherlands
}

Received 17 July 2003

Published 20 November 2003

Online at stacks.iop.org/JPhysCM/15/S3549

\begin{abstract}
We study, by simulation, the effect of attractive triplet interactions on the phase behaviour of suspensions of charged colloidal particles at low ionic strength, i.e. in the regime where the pair interactions are purely repulsive. We use the pair and triplet interactions that were obtained recently from a numerical, nonlinear Poisson-Boltzman study (Russ et al 2002 Phys. Rev. E 66 011402). Our simulations tentatively explain, for the first time, experimental observations of a rather large density difference between coexisting body centred cubic and face centred cubic crystal phases.
\end{abstract}

(Some figures in this article are in colour only in the electronic version)

\section{Introduction}

Charge-stabilized colloidal suspensions are extremely asymmetric fluid mixtures that consist of highly charged colloidal particles immersed in a molecular solvent with cations and anions. Such suspensions are abundant in industry (e.g. ink, paint) and biology (e.g. in cells) but, from a condensed matter point of view, their interest stems mainly from the fact that these multicomponent fluids can be regarded as one-component fluids of colloids interacting through tunable effective potentials. The standard and very successful effective one-component description of suspensions of charged colloids is due to Derjaguin, Landau, Verwey and Overbeek (DLVO) [1]. The DLVO theory predicts that the effective potential between two charged colloids at separation $r$ consists of a sum of:

(i) a hard-core repulsion due to the finite diameter $\sigma$ of the colloids;

(ii) van der Waals attractions with a typical range of a few nanometres beyond the colloidal surface; and 
(iii) a screened-Coulomb (Yukawa) repulsion $\propto \exp [-\kappa r] / r$, where $\kappa=\left(8 \pi e^{2} c_{\mathrm{s}} / \epsilon k T\right)^{1 / 2}$ is the inverse Debye screening length given in terms of the elementary charge $e$, the concentration $2 c_{\mathrm{s}}$ of monovalent anions and cations, the dielectric constant $\epsilon$ of the solvent, and the thermal energy $k T$.

The electrostatic screening is performed by the salt ions, which form an oppositely charged diffuse cloud of thickness $\kappa^{-1}$ around the charged colloidal surfaces. For water at room temperature, with the physically accessible range of salt concentrations $10^{-7} \mathrm{M} \lesssim c_{\mathrm{s}} \lesssim 10 \mathrm{M}$, it follows that $\kappa^{-1}$ varies over four decades from $1 \AA$ to $1 \mu \mathrm{m}$ upon decreasing $c_{\mathrm{s}}$ from its highest to its lowest possible value. At the highest salt concentrations, $\kappa^{-1}$ is smaller than the range of the dispersion forces, which explains the flocculation instability of suspensions due to the irreversible aggregation of colloids [2]. At intermediate salt concentrations, say $c_{\mathrm{s}} \simeq 10^{-3}-10^{-1} \mathrm{M}$, the range of the electrostatic repulsions and the dispersion forces are similar, and their competition and interplay explain, for instance, the reversible vapour-liquid transition [3] observed in this regime. At typical low salt concentrations, $c_{\mathrm{s}} \lesssim 10^{-3} \mathrm{M}$, the van der Waals attractions are usually masked by the screened-Coulomb repulsions, since $\kappa^{-1}$ is then (much) larger than the range of the attractions. The repulsive long-range nature of the interactions in this regime can explain observations of stable face centred cubic (fcc) and body centred cubic (bcc) crystal phases at packing fractions as low as a few per cent [2]. For these reasons, and many more, the DLVO potential is a true cornerstone of colloid science.

It is important to recall, however, that explaining the behaviour of suspensions in terms of the DLVO potential involves the tacit assumption of pairwise additivity of the effective interactions, i.e. implicitly one assumes that the interaction Hamiltonian of $N$ colloids, with coordinates $\boldsymbol{r}_{i}(i=1, \ldots, N)$, takes the pairwise form $H_{2}=\sum_{i<j}^{N} v_{2}\left(r_{i j}\right)$, where $r_{i j}=\left|\boldsymbol{r}_{i}-\boldsymbol{r}_{j}\right|$. Heuristically, one argues that pairwise additivity of the effective interactions can only be a reasonable approximation if the range of the effective pair-potential $v_{2}(r)$ is small compared to the diameter $\sigma$ of the colloidal spheres, i.e. if $\kappa \sigma \gg 1$. By contrast, one expects important triplet and higher-order 'overlaps' of the ionic clouds, and hence manybody potentials, if $\kappa \sigma \lesssim 1$. For typical colloidal diameters this condition is realized in the extremely low-salt regime of $10^{-5} \mathrm{M} \lesssim c_{\mathrm{s}} \lesssim 10^{-7} \mathrm{M}$, where $\kappa^{-1} \simeq 100-1000 \mathrm{~nm}$ for water at room temperature. Recent measurements of radial distribution functions point indeed to the breakdown of pairwise additivity in this low-salt regime [4]. More surprisingly, perhaps, direct experimental evidence of the qualitative breakdown of pairwise additivity in this regime has in fact long been available in the literature [5-8], but has not received any detailed attention. This could be due to the fact that these studies 'merely' yield fluid, fcc and bcc phases, as predicted on the basis of the DLVO potential [9]. The evidence of the breakdown stems from the large magnitude of the difference in densities between the coexisting phases in fluid-solid and bcc-fcc equilibrium. If one characterizes this difference by the ratio $x(>1)$ of the two coexisting densities, one finds $x \simeq 3$ [5] and $x=1.5$ [6] for fluid-solid coexistence and $x \simeq 1.26$ [7] and $x=1.78$ [8] for bcc-fcc coexistence. To appreciate the large magnitude of these density jumps, one recalls that $x=1.1$ for the fluid-fcc density jump in the hard-sphere system, that any repulsive softness tends to decrease $x$ towards unity (a smaller jump) [10], that the density jump across the fcc-bcc transition in soft-sphere systems is so small that one usually does not bother to determine it, and that 'volume' terms (which can be seen as coarsegrained many-body potentials) tend to decrease density jumps in low-salt suspensions [11]. In this article we explain the observed large density differences between the coexisting phases in low-salt colloidal suspensions, for the first time, as a direct consequence of non-pairwise interactions. 


\section{Model and methods}

The simplest possible non-pairwise correction to the effective Hamiltonian $H_{2}$ involves the triplet potential $v_{3}$, and the resulting interaction Hamiltonian is of the form

$$
H_{23}=\sum_{i<j}^{N} v_{2}\left(r_{i j}\right)+\sum_{i<j<k}^{N} v_{3}\left(r_{i j}, r_{i k}, r_{j k}\right) \text {. }
$$

Recently, $v_{2}$ and $v_{3}$ were calculated numerically within nonlinear Poisson-Boltzman theory for a model system of uniformly charged spheres (of total charge $Z e$, with $Z \gg 1$ ) immersed in a structureless solvent (with dielectric constant $\epsilon$, inverse temperature $\beta=1 / k T$, Bjerrum length $\lambda_{\mathrm{B}}=\beta e^{2} / \epsilon$, and Debye screening length $\kappa^{-1}$ ) [12]. It was found that $v_{2}$ (repulsive) and $v_{3}$ (attractive) can be represented accurately by

$$
\begin{aligned}
& \beta v_{2}(r)=A_{2} \frac{\sigma}{\lambda_{\mathrm{B}}} \frac{\exp [-\kappa r]}{r / \sigma}, \quad r>\sigma, \\
& \beta v_{3}\left(r_{i j}, r_{i k}, r_{j k}\right)=-A_{3} \frac{\sigma}{\lambda_{\mathrm{B}}} \frac{\exp \left[-\gamma\left(r_{i j}+r_{i k}+r_{j k}\right)\right]}{\left(r_{i j}+r_{i k}+r_{j k}\right) / \sigma},
\end{aligned}
$$

where $A_{2}, A_{3}$ and $\gamma$ are positive fit parameters which only depend on $\kappa \sigma$ and $Z \lambda_{\mathrm{B}} / \sigma \equiv \bar{Z}$, and which are tabulated extensively in [12]. These simple expressions for $v_{2}$ and $v_{3}$, which are consistent with the direct simulations of pair and triplet forces in systems of two and three colloids [13], enable us to perform efficient Monte Carlo simulations of $N$-body systems to study the effect of the attractive three-body potentials on the phase diagram of charge-stabilized suspensions. For Hamiltonians $H_{23}$ described by equation (1), we calculate the Helmholtz free energy of typically $N=250$ colloids in a volume $V$ for a range of densities $\rho=N / V$ by means of thermodynamic integration. We use the hard-sphere fluid and Einstein crystals as reference states for the fluid and the crystalline fcc and bcc phases, respectively, and perform common tangent constructions to determine phase coexistence [14]. Note that the counter-ion contribution to the Helmholtz free energy (or rather the grand potential) is correctly taken into account because the effective potentials $v_{2}$ and $v_{3}$ are based on an integration over the counter-ion's degrees of freedom [12].

\section{Results and conclusions}

We set $\lambda_{\mathrm{B}} / \sigma=0.0225$ and focus on calculating the phase behaviour for reduced charge $\bar{Z}=1.8$. For water at room temperature this corresponds to a colloidal charge of $Z=80$ and a diameter of $\sigma=32 \mathrm{~nm}$. As a benchmark we first calculate the phase diagram of the purely pairwise system, i.e. with $v_{3} \equiv 0$. Figure 1 shows the resulting phase diagram in the $\eta-\kappa \sigma$ representation, where $\eta=(\pi / 6) \rho \sigma^{3}$ is the colloidal packing fraction. Note that $\kappa$ does not depend on $\eta$. In fact, $\kappa$ is the screening parameter of a salt reservoir in osmotic contact with the colloidal suspension. The two-phase regions are grey, and tie lines between the coexisting phases are horizontal. The prefactors $A_{2}$ for $\kappa \sigma>2.56$ were obtained by linear extrapolation with respect to $\kappa \sigma$, as no numerical data were tabulated in this $\kappa \sigma$-regime [12]. The phase diagram shows a large bcc pocket below a fluid-bcc-fcc triple point at $\kappa \sigma \simeq 4.86$, and narrowing fluid-fcc, fluid-bcc and fcc-bcc coexistence regimes upon decreasing $\kappa \sigma$ (the density ratio $x \lesssim 1.03$ at all the binodals in the regime shown in figure 1 ). We find that the low- $\kappa \sigma$ part of the fluid-bcc coexistence line (i.e. that part where the hard-core is not expected to be important) is in good agreement with that of point-Yukawa particles presented in [15]. At high $\kappa \sigma$, beyond the scale of figure 1, the fluid-fcc coexistence line approaches that of hard spheres. 


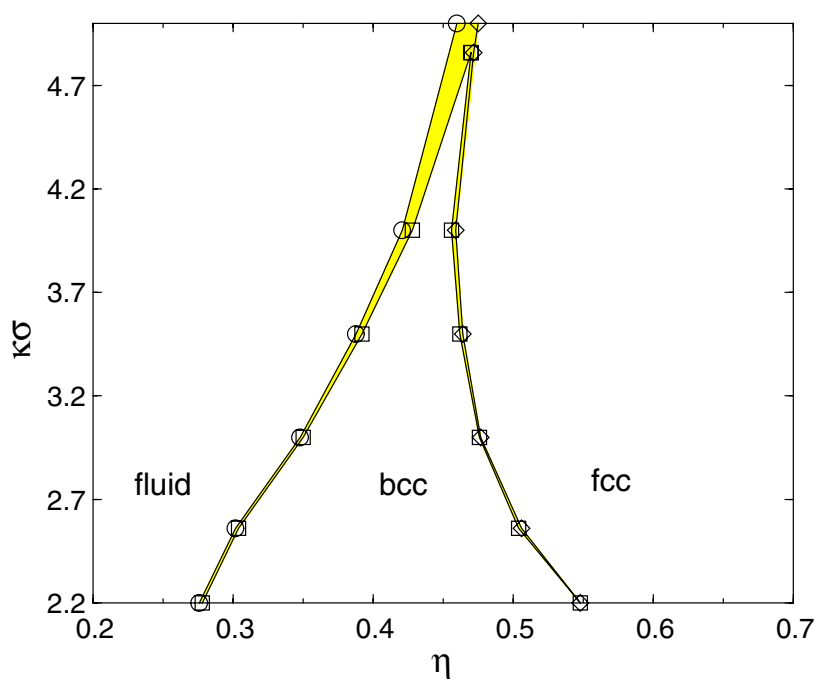

Figure 1. Phase diagram of a pairwise aqueous suspension of charged colloids (charge $Z=80$, diameter $\sigma=32 \mathrm{~nm}$, packing fraction $\eta$ ) in osmotic contact with a reservoir of monovalent salt ions (Debye length $\kappa^{-1}$ ), as obtained from simulation. We distinguish fluid, bcc, and fcc phases that are separated by (grey) two-phase regions merging in a triple point at $\kappa \sigma \simeq 4.8$. The two-phase regions represent relatively small density jumps, i.e. $x \leqslant 1.03$ (see text).

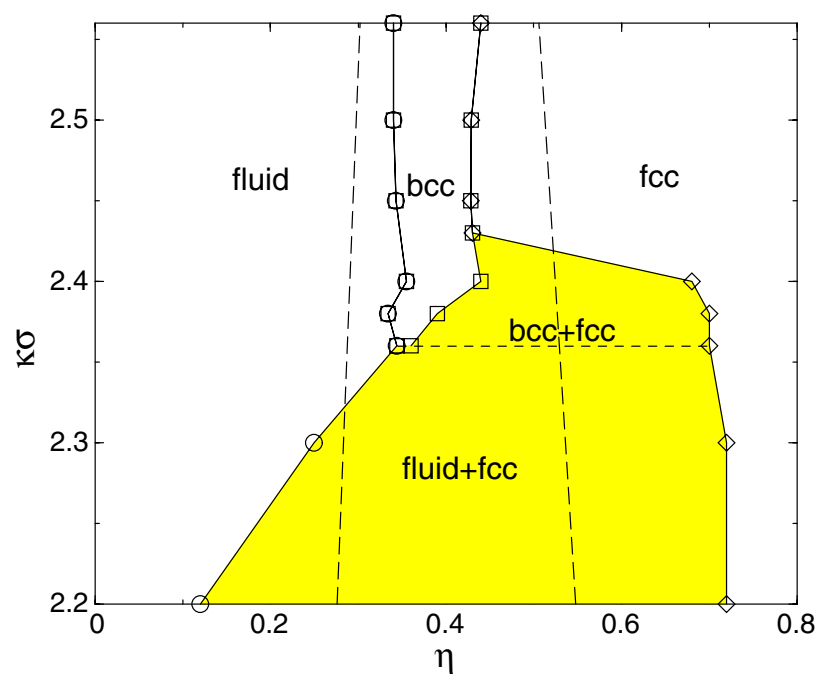

Figure 2. As figure 1, but now the Hamiltonian includes the triplet attractions, see equation (1). Note the different $\kappa \sigma$-scale. The grey areas represent two-phase coexistence, the horizontal dashed line is the lower fluid-bcc-fcc triple point, and the two almost vertical dashed lines represent the fluid-bcc and bcc-fcc phase boundaries of the pairwise system of figure 1 .

In figure 2 we present the phase diagram for the same parameters as in figure 1, but now including the triplet potential. Note the different $\kappa \sigma$-scale of figure 2; linear interpolation of the data of [12] was used to obtain the potentials in the required regime of $\kappa \sigma$. Figure 2 shows that an important effect of the inclusion of the triplet potential is the reduction of the stability range of the bcc phase, which is now not only confined by an upper triple point (at 
$\kappa \sigma \simeq 4.86$, as in the pairwise system) but also by a lower one at $\kappa \sigma \simeq 2.36$. Below the latter the system with triplet attractions exhibits a broad fluid-fcc coexistence (with $x \gtrsim 2$ ), whereas in the regime $2.36 \lesssim \kappa \sigma \lesssim 2.56$ a very narrow fluid-bcc coexistence occurs at $\eta \approx 0.35$ and a rather broad bcc-fcc coexistence occurs at higher $\eta$. The density ratio of the latter shrinks from $x \simeq 2$ at $\kappa \sigma=2.36$ to essentially unity (a negligible jump) at $\kappa \sigma \approx 2.43$. The essentially vertical dashed lines in figure 2 give the fluid-bcc and bcc-fcc phase boundaries of the pairwise system of figure 1. By comparing the Madelung energies of the pairwise and the pair-and-triplet system we estimate that the phase diagrams of figures 1 and 2 essentially coincide for $\kappa \sigma \gtrsim 4$, i.e. in the high-salt regime the effect of triplet forces is vanishingly small, as expected. We argue that there must be a regime in the $(\eta, \kappa \sigma)$ plane where the dominant correction to pairwise additivity is the triplet potential, but that higher-order terms will become important at sufficiently low $\kappa \sigma$ and high $\eta$. One expects that the onset of the broadening of bcc-fcc coexistence (at $\kappa \sigma \simeq 2.43$ ) is essentially an effect induced by the triplet attractions, but higher-order potentials will doubtlessly affect the dense fcc phase at $\eta \simeq 0.7$ at lower $\kappa \sigma$. In this sense our key result is the widening of the bcc-fcc coexistence at $\kappa \sigma \simeq 2.4$, rather than that of the fluid-fcc coexistence below the lower triple point. In fact, one could argue that the extremely dense coexisting fcc phase for $\kappa \sigma \lesssim 2.36$ at $\eta \simeq 0.7$ is an unrealistic artifact of the truncation of the effective Hamiltonian beyond the triplet terms, which would be restored to, say, $\eta \simeq 0.5$ if sufficient higher-body terms were taken into account. Given the fact that the four-body potential is also of an attractive nature [12], at least for those few configurations for which it was calculated, one expects even broader two-phase regions if the truncation is performed after the four-body term. In other words, one would have to include terms up to $v_{5}$ (or even higher, if $v_{5}$ also turns out to be attractive) in order to shift the coexisting fcc density to $\eta \simeq 0.5$. This is a rather unattractive perspective, and indicates perhaps that the systematic expansion of the effective Hamiltonian into density-independent $n$-body potentials is not efficient enough to produce quantitative predictions in the regime of low $\kappa \sigma$.

We now wish to compare the phase diagram of figure 2 with the available experimental phase diagrams of $[5,7,8]$. At first sight such a direct comparison seems impossible, since the present calculations are based on the dimensionless combinations $\bar{Z}=1.8$ and $\lambda_{\mathrm{B}} / \sigma=0.0225$, which do not coincide with those of the experiments, where $\bar{Z}^{\prime}=2.16$ and $\lambda_{\mathrm{B}}^{\prime} / \sigma^{\prime}=0.016$ in the low-salt regime of [7] and $\bar{Z}^{\prime} \simeq 4-5$ and $\lambda_{\mathrm{B}}^{\prime} / \sigma^{\prime} \simeq 0.005-0.006$ in $[5,8]$. Here (and below) the primes denote experimental values, where the experimental charge number $Z^{\prime}$ is based on the assumption that the pair interaction is of the DLVO form $\beta v_{\mathrm{DLVO}}(r)=\left(Z^{\prime} /\left(1+\kappa^{\prime} \sigma^{\prime} / 2\right)\right)^{2} \lambda_{\mathrm{B}}^{\prime} \exp \left[\kappa^{\prime}\left(\sigma^{\prime}-r\right)\right] / r$. Yet even though the simulated system differs from the experimental ones, it is possible to construct a mapping $(\eta, \kappa \sigma) \rightarrow\left(\eta^{\prime}, \kappa^{\prime} \sigma^{\prime}\right)$ that, arguably, relates the simulated system to an experimental one, albeit approximately. The mapping is based on the equality of the pair-potential $v_{2}$ of equation (2) used in the simulations and the experimental DLVO potential $v_{\text {DLVO }}$, at equal scaled separation $s=r / a$ and $r / a^{\prime}$, respectively, where $a=\rho^{-1 / 3}$ is the typical interparticle spacing in the simulations and $a^{\prime}$ is that in the experiment. This condition can be written as $v_{2}(a s)=v_{\text {DLVO }}\left(a^{\prime} s\right)$ for all $s$ sufficiently large, and the equality of the exponential decay yields $\kappa a=\kappa^{\prime} a^{\prime}$ or, equivalently,

$$
\eta^{\prime}=\frac{\left(\kappa^{\prime} \sigma^{\prime}\right)^{3}}{(\kappa \sigma)^{3}} \eta
$$

The required $\kappa^{\prime} \sigma^{\prime}$ follows from the condition of equal prefactors:

$$
\frac{\exp \left(\kappa^{\prime} \sigma^{\prime}\right) \kappa^{\prime} \sigma^{\prime}}{\left(1+\kappa^{\prime} \sigma^{\prime} / 2\right)^{2}}=\frac{A_{2}}{\left(Z^{\prime}\right)^{2}} \frac{\sigma}{\lambda_{\mathrm{B}}} \frac{\sigma^{\prime}}{\lambda_{\mathrm{B}}^{\prime}} \kappa \sigma,
$$

which uniquely maps $\kappa \sigma$ to $\kappa^{\prime} \sigma^{\prime}$ for fixed $Z^{\prime}, \lambda_{\mathrm{B}}^{\prime} / \sigma^{\prime}$ and $\lambda_{\mathrm{B}} / \sigma$ (recall that $A_{2}=A_{2}(\kappa \sigma, \bar{Z})$ ). The equations (4) and (5) constitute the mapping that we propose here. 


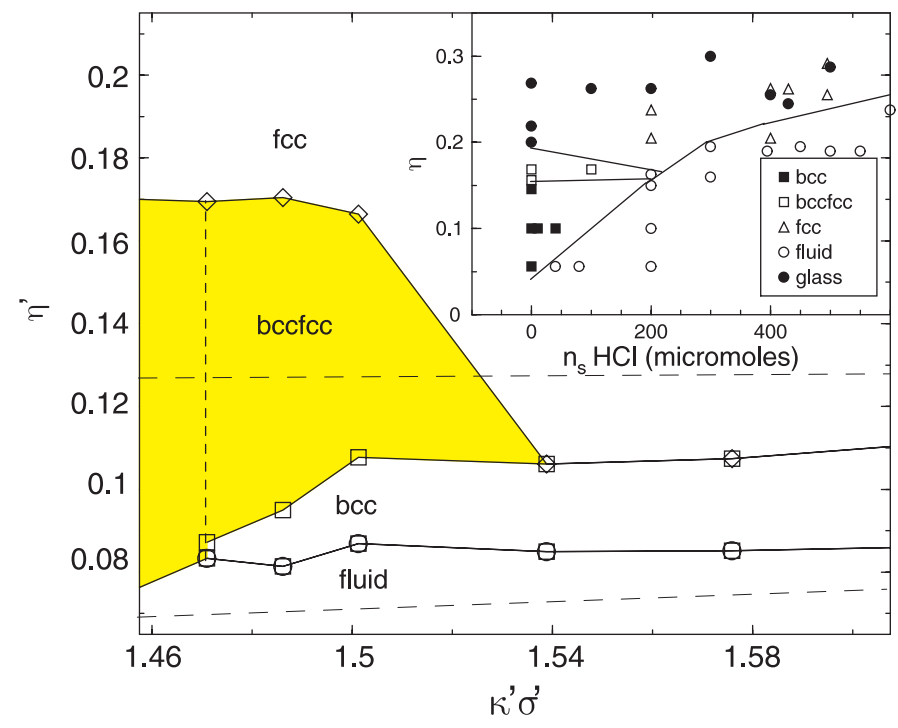

Figure 3. A mapping of figure 2 (see text) on the experimental conditions of [7]. The inset shows the experimental phase diagram of [7]. The symbols are as in figures 1 and 2 .

We note that a better comparison with the experimental phase diagram would be based on repeating the calculation with the value of $\bar{Z}$ used in the experiments, instead of using the mapping given by equations (4) and (5). However, given that the calculation of the phase diagram in figure 2 requires about one year of CPU time, we cannot simply re-calculate it for a larger parameter set at this stage.

Before comparing the results of the mapping represented by the equations (4) and (5), we wish to stress that mapping state points on the basis of the equality of scaled pair-potentials is bound to suffer from several short-comings. For instance, in this mapping we ignored the hard-core contribution of the two pair-potentials-equating these in the scaled systems would yield $\sigma / a=\sigma^{\prime} / a^{\prime}$, i.e. $\eta=\eta^{\prime}$. The mapping can therefore only be quantitative for state-points where the soft repulsions are strong enough to prevent contact. Another shortcoming is caused by the fact that the mapping ignores the triplet potential. However, this is harmless if the triplet potentials of two systems (primed and unprimed) satisfy the same scaling as the pair interactions, i.e. $v_{3}\left(\left\{a s_{i j}\right\}\right)=v_{3}^{\prime}\left(\left\{a^{\prime} s_{i j}\right\}\right)$ where $s_{i j}$ are the scaled particle separations. For interactions of the form of equations (2) and (3), the simultaneous scaling of pair and triplet potentials can be satisfied provided that $\gamma / \kappa$ and $A_{2} / A_{3}$ are constants that are independent of $\kappa \sigma$ and $\bar{Z}$. Although one checks from the tabulated values of [12] that this condition is not met exactly, one observes that the aforementioned ratios vary by, say, a factor 2-3, whereas $A_{2}$ etc individually vary over one to two decades for $\bar{Z} \geqslant 1.8$. In other words, the pair and triplet interactions scale in an approximately similar fashion, which lends the mapping at least some qualitative credibility. A very bona fide aspect of the mapping is that $\kappa^{\prime} \sigma^{\prime}$ depends only on $\kappa \sigma$ and not on $\eta$ (see equation (5)) and, as a consequence, any tie-line at a fixed $\kappa \sigma$ is mapped onto another one at some fixed $\kappa^{\prime} \sigma^{\prime}$.

In figure 3 we present the result of the mapping of the phase diagram of figure 2 using equations (4) and (5) and the experimental parameters $Z^{\prime}$ and $\lambda_{\mathrm{B}}^{\prime} / \sigma^{\prime}$ given by Sirota et al [7]; the inset shows the corresponding experimental phase diagram [7]. Taking into account that no free parameters are used, it is fair to say that there is some notable agreement, structurally as well as numerically. For instance, both phase diagrams of figure 3 have packing fractions and 
Debye screening lengths in the same regime (Sirota et al give $\kappa^{\prime} \sigma^{\prime}=2.3$ at $\eta^{\prime}=0.10$ for their low-salt regime). Regarding the structure, we note that at $\kappa^{\prime} \sigma^{\prime} \approx 1.5$ (i.e. low salt concentration in Sirota et al) both phase diagrams exhibit, upon increasing density, a sequence of fluid-bccfcc (or glass), with a small density jump at the fluid-bcc transition and a considerable jump at the bcc-fcc (or glass) transition. While the order of occurrence of the bcc and fcc phases would also be predicted correctly by a calculation based on only two-body interactions, such calculations would certainly not predict the large density difference between coexisting bcc and fcc crystals. At higher salt concentrations (above $200 \mu \mathrm{M}$ ), the phase diagram of Sirota et al has a fluid-bcc-fcc triplet point beyond which no bcc pocket is seen. Though not shown in figure 3, our phase diagram also has this triple point, and it is estimated to be around $\kappa^{\prime} \sigma^{\prime} \simeq 3.37$. When comparing the values of $\kappa \sigma$ in our calculations with those of Sirota $e t a l$, it is important to recall that our $\kappa \sigma$ is that of the salt reservoir while, in the experiments, $\kappa \sigma$ also includes the screening due to the counter-ions. This makes a direct comparison with experimental data particularly difficult.

There are also apparent discrepancies in the two phase diagrams in figure 3. The first is that, in the low-salt regime, our phase diagram does not predict a stable bcc phase, while it is clearly present in the experiments. However, we argue that, in the very-low-salt regime, the applicability of the many-body potential that is truncated after the triplet term loses its validity, i.e. four- and higher-body terms become important. Second, the agreement is by no means quantitative: the coexisting packing fractions of the two phase diagrams are in the same regime but not identical, and our calculations predict broader coexistence than is seen in the experiments. As already mentioned earlier, we expect that the coexistence would become narrower if many more $n$-body terms were considered, but this is rather impractical. The purpose of our calculations was to study the effect of the three-body interactions as a first-order correction to the pairwise picture. It is unrealistic to expect that such a first-order correction yields quantitatively accurate results down to very-low-salt concentrations.

In the same fashion, we also attempted to map the phase diagram of figure 2 on those of $[5,8]$. For both cases we could only obtain quantitative agreement if we reduced the reported colloidal charges considerably. For instance, if we set $Z^{\prime}=480$ instead of the reported $Z^{\prime}=700$ [8], we find that the bcc-fcc coexistence region extends from $\eta^{\prime}=0.0026 \pm 0.0004$ to $\eta^{\prime}=0.0045 \pm 0.0001$ at $\kappa^{\prime} \sigma^{\prime} \approx 0.45$. This matches the 'salt-free' observations of [8] quantitatively. Similarly, if we set $Z^{\prime}=350$ instead of $Z^{\prime}=880$ as reported in [5], the fluid-fcc coexistence is in the regime from $\eta^{\prime}=0.010 \pm 0.005$ to $\eta^{\prime}=0.030 \pm 0.004$ at $\kappa^{\prime} \sigma^{\prime} \approx 0.8$, as observed experimentally in this low-salt regime [5]. Interestingly, it seems that another theoretical attempt to reproduce the experimental low-salt freezing line of [5] also needs colloidal charges much lower than the reported ones [16].

We conclude that triplet attractions can explain, at least qualitatively, some of the large density differences that are observed experimentally between coexisting phases in low-salt suspensions of highly charged colloidal spheres.

\section{Acknowledgments}

It is a pleasure to thank $\mathrm{C}$ Russ and $\mathrm{H}$ von Grünberg for stimulating discussions. This work is part of the Research program of the 'Stichting voor Fundamenteel Onderzoek der Materie (FOM)', which is supported financially by the 'Nederlandse Organisatie voor Wetenschappelijk Onderzoek (NWO)'. We also thank the Dutch National Computer Facilities foundation for access to the SGI Origin3800. The High Performance Computing group of Utrecht University is gratefully acknowledged for ample computer time. A-P H also gratefully acknowledges financial support from the Finnish Cultural Foundation. 


\section{References}

[1] Derjaguin B and Landau L 1941 Acta Physicochim. USSR 14633

Verwey E J W and Overbeek J Th G 1948 Theory of the Stability of Lyotropic Colloids (Amsterdam: Elsevier)

[2] Russel W B, Saville D A and Schowalter W R 1989 Colloidal Dispersions (Cambridge: Cambridge University Press)

[3] Victor J M and Hansen J-P 1985 Trans. Faraday Soc. 8143

[4] Brunner M, Bechinger C, Strepp C W, Lobaskin V and von Grünberg H H 2002 Europhys. Lett. 58926 Klein R, von Grünberg H H, Bechinger C, Brunner M and Lobaskin V 2002 J. Phys.: Condens. Matter 147631

[5] Monovoukas Y and Gast A P 1989 J. Colloids Interface Sci. 128533

[6] Philipse A P and Koenderink G H 2003 Adv. Colloid Interface Sci. 100-102 613

[7] Sirota E B, Ou-Yang H D, Sinha S K, Chaikin P M, Axe J D and Fujii Y 1989 Phys. Rev. Lett. 621524

[8] Schöpe H J, Decker T and Palberg T 1998 J. Chem. Phys. 10910068

[9] Salgi P and Rajagopalan R 1991 Langmuir 71383

Sengupta S and Sood A K 1991 Phys. Rev. A 441233

Choudhury N and Gosh S K 1995 Phys. Rev. E 514503

[10] Pollock E L and Hansen J-P 1973 Phys. Rev. A 83110

[11] Graf H and Löwen H 1998 Phys. Rev. E 575744

[12] Russ C, von Grünberg H H, Dijkstra M and van Roij R 2002 Phys. Rev. E 66011402

[13] Löwen H and Allahyarov E 1998 J. Phys.: Condens. Matter 104147

[14] Frenkel D and Smit B 2002 Understanding Molecular Simulations 2nd edn (London: Academic)

[15] Hamaguchi S, Farouki R T and Dubin D H E 1997 Phys. Rev. E 564671

[16] Trizac E, Bocquet L and Aubouy M 2002 Phys. Rev. Lett. 89248301 